\title{
ARTICLE
}

\section{Systematic significance of micromorphological and palynologi- cal characteristics in Lagochilus Bunge ex Benth. (Lamiaceae) in Iran}

\author{
Fatemeh Azimishad ${ }^{1 *}$, Masoud Sheidai ${ }^{1}$, Maryam Keshavarzi ${ }^{2}$, Seyed Mehdi Talebi ${ }^{3}$, \\ Zahra Noormohammadi ${ }^{4}$
}

${ }^{1}$ Faculty of Life Sciences and Biotechnology, Shahid Beheshti University, Tehran, Iran

${ }^{2}$ Department of Plant Sciences, Faculty of Biological Science, Alzahra University, Tehran, Iran

${ }^{3}$ Department of Biology, Faculty of Sciences, Arak University, Arak 38156-8-8349, Iran

${ }^{4}$ Biology Department, Islamic Azad University, Sciences and Research Branch, Tehran, Iran

\begin{abstract}
Lagochilus is a genus with ten taxa (species, subspecies and variety) in Iran, which nine of them are endemic. This is the first micromorphological investigation of this genus. Micromorphological features of trichomes on the stems, leaves and calyces, and also pollen morphology of 19 populations were investigated by scanning electron microscopy. Two types of trichome (glandular and non-glandular) including 14 forms were described. Here, among the non-glandular trichomes, cell number and size of trichomes are considered as valuable characteristics, while the glandular trichomes are observed as stalked, sessile and peltate. Lagochilus pollen grains are tricolpate and small to medium sized. The basic shape of the pollen grains in most taxa is prolate, however prolate-subprolate pollen grains was recorded for L. aucheri ssp. aucheri var. aucheri 2. Four types of exine sculpture patterns were distinguished: bireticulate, reticulate, microreticulate and incomplete reticulate. Quantitative and qualitative characteristics were examined by multivariate analysis. The results indicated that the studied taxa were separated from each other; however varieties of $L$. aucheri did not grouped together. The results support the existence of known varieties in L. aucheri. In general, our investigations reveal the usefulness of micromorphological characteristics in taxon delimitation at the specific and infraspecific levels.

Acta Biol Szeged 63(2):143-155 (2019)
\end{abstract}

\author{
KEY WORDS \\ Lagochilus \\ Lamiaceae \\ SEM \\ trichome micromorphology \\ palynology
}

\section{ARTICLE INFORMATION}

Submitted

29 December 2019

Accepted

03 March 2020

*Corresponding author

E-mail: ailarazimishad3@gmail.com

\section{Introduction}

The genus Lagochilus Bunge ex Benth. (1834), belong to family Lamiaceae, subfamily Lamioideae (Bendiksby et al. 2011), comprises about 44 species, from which six species occur in Iran (Jamzad 1988; Zhang et al. 2017). Lagochilus species are mostly distributed in dry slopes, valleys and deserts from Iran to Mongolia, Russia (south Siberia), northwest China and north Pakistan, but have wide distribution in Central Asia (Harley et al. 2004; Zhang et al. 2017).

The chemical components of several Lagochilus species were surveyed due to their medicinal uses. Lagochilin is a diterpene which was found in various species of the genus, and is thought to be responsible for the sedative, hypotensive and hemostatic effects of these species (Chizhov et al. 1970; Jiao et al. 2014). Taban et al. (2009) have reported the antibacterial activity of flowers and leaves of L. kotschyanus Boiss. (1848) oils against four
Gram-positive and two Gram-negative bacteria.

These plants are subshrubs or perennial herbs with woody root and green-white, rigid stem with spiny bracteoles. Leaf blade is rhombic, palmatipartite or pinnatipartite with campanulate-tubular calyx and villous-pillose corolla. Nutlets flattened-obconical, oblong-obovoid or oblongovid (Jamzad 1988).

Ikramov (1976) investigated the classification, distribution, and community ecology of Lagochilus species in Central Asia of former USSR. Recently, Zhang et al. (2017) have studied Lagochilus species using chloroplast sequence data to reveal the species relationships as well as the date of divergence. However only three species sampled from Iran was included in their investigation and they were considered as a complex and very much different clade within the genus Lagochilus (Zhang et al. 2017).

Boissier (1879) mentioned 6 species, Parsa (1949) listed 6 species and two varieties and Rechinger (1982) recognized 5 species from Iran. Jamzad (1988) identified 6 species through revision of the genus Lagochilus in Iran, these 
are L. alutaceus Bunge (1873), L. aucheri Boiss. (1844), L. lasiocalyx (Stapf) Jamzad (1988), L. macranthus Fisch. \& C.A. Mey. (1841), L. quadridentatus Jamzad (1988) and L. cabulicus Benth. (1848). The first five species are endemic to Iran and are considered as the endangered species (Jalili and Jamzad 1999).

Jamzad (1988) described L. quadridentatus and 3 infraspecific taxa as L. aucheri ssp. heterophyllus, L. aucheri ssp. aucheri var. elegans and L. aucheri ssp. aucheri var. tomentosus. She found a complexity in naming of L. aucheri group. Morphological characteristics as calyx teeth number, calyx width, shape of leaves and indumentum type of different parts of calyx, nutlet and stem traits were used in identification key for specific and infraspecific level. However, according to the Plant List, there are five species in Iran and known subspecies and varieties by Jamzad are synonym names (Govaerts et al. 2013).

The taxonomic value of trichomes density and types and also pollen morphology in identification, delimitation and phylogenetic reconstruction of some members of the family Lamiaceae have been elucidated by many authors (Erdtman 1945; Wunderlich 1967; Abu-Asab and Cantino 1989; 1992; 1993a,b; 1994; Cantino 1990; Doaigey1991; Bini Maleci and Servettaz, 1991; Harley et al. 1992; Wagstaff 1992; Navarro and El Qualidi, 2000; Moon and Hong 2003; C,elenk et al. 2008 a,b; Moon et al. 2008a,b,c; Salmaki et al. 2008; Hassan et al. 2009; Gairola et al. 2009; Salmaki et al. 2009; Özler et al. 2011; 2013; Firdous et al. 2015 ; Atalay et al. 2016a,b; Talebi et al. 2018a,b). Besides of systematic significance, trichomes have several roles in plants. For example, there are assumed to attraction of pollinators (Wagner 1991), protection from herbivores and pathogens (Xiao et al. 2017) and decrease the leaf temperature and transpiration (Peter and Shanower 1998).

Pollen morphology is generally supported segregation of some genera of Lamiaceae (Abu-Asab and Cantino 1994). Palynological study of the genus Lagochilus have been poorly investigated and is still lacking (Abu-Asab and Cantino 1994; Talebi and Rezakhanlou 2012; Badamtsetseg et al. 2012), so their work comprises only a few Lagochilus species.

Whereas phylogenetic and molecular studies of most Lagochilus species have not been investigated yet and also there is no comprehensive micromorphological and palynological study covering all species of Lagochilus in Iran and Asia, therefore, the main objectives of the present study are to provide a detailed investigation and description of trichomes and pollen micromorphology of the genus Lagochilus, mainly by scanning electron microscopy (SEM), to determine whether these data can be of value in the taxonomy of the genus and delimitation of the species. Furthermore, trichomes and pollen morphology of most species was described for the first time.

Table 1. Voucher specimens of the genus Lagochilus examined in the present study. Ns: number of plant samples.

\begin{tabular}{|c|c|c|c|c|c|c|}
\hline Taxon & Ns & Locality & Latitude & Longitude & Altitude (m) & Voucher code \\
\hline L. aucheri ssp. aucheri var. aucheri 2 & 5 & Zanjan, Dash kasan Mt. & $36^{\circ} 43^{\prime} 58^{\prime \prime}$ & $48^{\circ} 79^{\prime} 20^{\prime \prime}$ & 1700 & HSBU2018650 \\
\hline L. aucheri ssp. aucheri var. aucheri 2 & 5 & Zanjan, 90 km of Zanjan-Bijar road & $35^{\circ} 75^{\prime} 79^{\prime \prime}$ & $48^{\circ} 48^{\prime} 22^{\prime \prime}$ & 1500 & HSBU2018651 \\
\hline L. aucheri ssp. aucheri var. aucheri 2 & 5 & Zanjan, Bulamaji village & $36^{\circ} 56^{\prime} 31^{\prime \prime}$ & $48^{\circ} 48^{\prime} 79^{\prime \prime}$ & 1500 & HSBU2019100 \\
\hline L. aucheri ssp. aucheri var. aucheri 1 & 5 & Qazvin, Abegarm & $35^{\circ} 80^{\prime} 03^{\prime \prime}$ & $49^{\circ} 31^{\prime} 19^{\prime \prime}$ & 1500 & HSBU2018652 \\
\hline L. aucheri ssp. aucheri var. aucheri 3 & 5 & Alborz, Karaj, Dizin & $35^{\circ} 82^{\prime} 00^{\prime \prime}$ & $50^{\circ} 97^{\prime} 00^{\prime \prime}$ & 2500 & HSBU2018653 \\
\hline L. aucheri ssp. aucheri var. tomentosus & 5 & Tehran, Firuzkuh, Sarbandan & $35^{\circ} 75^{\prime} 50^{\prime \prime}$ & $52^{\circ} 77^{\prime} 24^{\prime \prime}$ & 2000 & HSBU2018654 \\
\hline L. aucheri ssp. aucheri var. tomentosus & 5 & Tehran, Damavand, Voleyran & $35^{\circ} 68^{\prime} 84^{\prime \prime}$ & $52^{\circ} 06^{\prime} 38^{\prime \prime}$ & 2000 & HSBU2019101 \\
\hline L. aucheri ssp. aucheri var. tomentosus & 3 & Tehran, Damavand, Tar lake & $35^{\circ} 73^{\prime} 17^{\prime \prime}$ & $52^{\circ} 21^{\prime} 00^{\prime \prime}$ & 1950 & HSBU2019102 \\
\hline L. aucheri ssp. aucheri var. kotschyanus & 4 & Markazi, Delijan & $34^{\circ} 06^{\prime} 38.7^{\prime \prime}$ & $50^{\circ} 32^{\prime} 23^{\prime \prime}$ & 1511 & HSBU2018658 \\
\hline L. aucheri ssp. aucheri var. kotschyanus & 4 & Markazi, Mahallat & $34^{\circ} 00^{\prime} 43.2^{\prime \prime}$ & $50^{\circ} 32^{\prime} 14.2^{\prime \prime}$ & 1910 & HSBU2019103 \\
\hline L. aucheri ssp. aucheri var. elegans & 5 & Qazvin, Takestan & $36^{\circ} 07^{\prime} 21^{\prime \prime}$ & $49^{\circ} 70^{\prime} 13^{\prime \prime}$ & 1450 & HSBU2018660 \\
\hline L. alutaceus & 5 & Razavi Khorasan, Chenaran & $36^{\circ} 30^{\prime} 52.3^{\prime \prime}$ & $59^{\circ} 02^{\prime} 42^{\prime \prime}$ & 1450 & HSBU2018655 \\
\hline L. cabulicus & 5 & North Khorasan, Esfarayen & $36^{\circ} 56^{\prime} 0.23^{\prime \prime}$ & $57^{\circ} 44^{\prime} 3^{\prime \prime}$ & 1535 & HSBU2018656 \\
\hline L. cabulicus & 5 & Razavi Khorasan, Chenaran & $36^{\circ} 30^{\prime} 52.3^{\prime \prime}$ & $59^{\circ} 02^{\prime} 42^{\prime \prime}$ & 1450 & HSBU2018661 \\
\hline L. macracanthus & 5 & Alborz, Eshtehard, Jafar abad village & $35^{\circ} 72^{\prime} 44^{\prime \prime}$ & $50^{\circ} 36^{\prime} 62^{\prime \prime}$ & 1300 & HSBU2018657 \\
\hline L. macracanthus & 5 & Alborz, Eshtehard, Nekujar village & $35^{\circ} 67^{\prime} 84^{\prime \prime}$ & $50^{\circ} 39^{\prime} 29^{\prime \prime}$ & 1450 & HSBU2019104 \\
\hline L. macracanthus & 5 & Tehran, Vardavard Mt. & $35^{\circ} 73^{\prime} 60^{\prime \prime}$ & $51^{\circ} 10^{\prime} 30^{\prime \prime}$ & 1500 & HSBU2019105 \\
\hline L. macracanthus & 4 & 20-kilometer after Saveh, Samavak village. & $35^{\circ} 03^{\prime} 85^{\prime \prime}$ & $50^{\circ} 08^{\prime} 42^{\prime \prime}$ & 1500 & HSBU2019106 \\
\hline L. lasiocalyx & 4 & Isfahan, Bagherabad & $33^{\circ} 51^{\prime} 12.4^{\prime \prime}$ & $50^{\circ} 33^{\prime} 9^{\prime \prime}$ & 1638 & HSBU2018659 \\
\hline
\end{tabular}




\section{Materials and Methods}

\section{Plant samples}

Totally 19 accessions of 10 taxa (species, subspecies and variety) were examined. In each populations, three to five plants were sampled for the scanning electron microscopy (SEM). Vouchers were collected from nature (2016-2018) and identified based on the descriptions provided in Flora Iranica (Rechinger 1982) and Flora of Iran (Jamzad 2012). Accessions studied and voucher details are listed in Table 1. All vouchers of the current study were deposited in Herbarium of Shahid Beheshti University (HSBU).

\section{Micromorphological characters}

Micromorphological features of leaves, stems and calyces were assessed using SEM. One to five mature leaves, stems and calyces were selected from fresh plant materials from each population. Middle parts of the samples were mounted directly on aluminum stubs using double-sided adhesive tape. The samples were coated with gold by a magnetron, sputtering device for about 10 nanometer thickness and observed with a SU 3500 SEM (Hitachi).

The terminology used is based on Cantino (1990) and Navarro and El Qualidi (2000) for trichomes. Size measurements on SEM images were made using UTHSCSA Image Tool Ver.3.0. Trichomes density was calculated by dividing the hair number per $\mathrm{mm}^{2}$ by the samples area (Gonzales et al. 2008). The distribution and types of trichomes were determined (Table 2).

\section{Pollen grains}

Fully matured anthers were obtained from the specimens and were prepared for study by SEM. Pollen grains were placed directly to aluminum stubs with double-sided cellophane tape and coated with gold by a magnetron sputtering device for about $10 \mathrm{~nm}$ thickness, then observed in a Hitachi SU 3500 SEM (in the Central Laboratory of Shahid Beheshti University) at $15 \mathrm{kV}$ and photographed at magnifications ranging from $\times 600$ to $\times 10,000$ to determine the exine ornamentation. Fully developed pollen grains (15-30 pieces) were selected and the following pollen measurements were taken: polar axis $(\mathrm{P})$, equatorial axis (E), colpus length (Clg), mesocolpium thickness, apocolpium diameter and $\mathrm{P} / \mathrm{E}$ ratio (Table 3 ). All of the measurements were performed using UTHSCSA Image Tool Ver. 3.0. The pollen terminology follows that of used by Faegri and Iversen (1975), Abu-Asab and Cantino (1994) and Hesse et al. (2009).

\section{Statistical analysis}

For multivariate analysis, the mean of quantitative characteristics was used, while qualitative characters were coded as binary/multistate features. Standardized vari- ables (mean $=0$, variance $=1$ ) were used in the statistical analysis. For grouping the studied taxa, cluster analysis using UPGMA (Unweighted Paired Group with Arithmetic Average) methods was performed using Euclidean and taxonomic distance among the species. Principal Coordinate Ordination (PCO) and Principal Coordinate Analysis (PCA) were performed (Podani 2000). Moreover, the one-way analysis of variance (ANOVA) was used to compare the trichomes numbers among the studied species. PAST (Paleontological statistics software package) version 2.17 was used for statistical analysis (Podani 2000).

\section{Results}

Different trichome features as trichome type, size and density on the stems, leaves and calyces of the studied taxa along their palynological data are summarized in Tables 2 and 3, respectively. Selected SEM micrographs of different trichome types and pollen grains studied were presented in Figs. 1-3 and Figs. 4-6, respectively.

\section{Micromorphological characters}

In general, two main types of trichomes were observed in the studied taxa: non-glandular (NG) and glandular (G). Both non-glandular and glandular trichomes occur on the same organ, particularly in the leaves and calyces.

\section{Non-glandular trichomes}

Non-glandular type trichomes were simple and unbranched in the examined specimens. However, this type varied in density, size, cell number, thickness of cell wall and presence of papillae on the trichome surface, therefore they were classified into 4 types (A-D, Table 2 ):

\section{Unicellular trichomes $(A)$.}

A1: prickles-hairs. These were the unicellular epidermal appendages. In terms of size, this form was shorter than $50 \mu \mathrm{m}$ on the leaves of L. cabulicus, L. macracanthus and L. aucheri ssp. aucheri var. elegans (Fig. 1A-B) to $200 \mu \mathrm{m}$ in the leaves and calyces of most specimens (except $L$. aucheri ssp. aucheri var. aucheri 3), which have papillate surface (Fig. 1C). The highest number of this kind of trichomes was seen on the leaves of L. aucheri ssp. aucheri var. tomentosus and its lowest amount was found on the leaves of $L$. macracanthus and $L$. aucheri ssp. aucheri var. aucheri 1 (Table 2).

A2: thin-walled trichomes. This form was triangular and very thin-walled hairs with ridges. In the term of size, thin-walled non-glandular trichomes can be subdivided into two types; short (i): shorter than $50 \mu \mathrm{m}$, which was found on the stems and calyces of L. macracanthus and L. cabulicus (Fig 1D) to hairs ranged from 50-300 $\mu \mathrm{m}$ on 
Azimishad et al.

Table 2. Indumentum characteristics of the Lagochilus taxa examined. Numbers refer to mean \pm standard deviation of trichomes number. NG: non-glandular. A1: prickles-hairs. A2-i: short thin-walled trichomes. A2-ii: long thin-walled trichomes. B1: short bicelled trichomes densely covered by micropapillae. B2: short bicelled trichomes, the basal cell is without micropapillae. B3: long bicelled trichomes. C: long tricelled trichomes. D: long four-celled trichomes. G: glandular. G1: sessile capitate. G2: short-stalked capitate. G3: peltate.

\begin{tabular}{|c|c|c|c|c|c|c|c|c|c|c|c|c|c|c|}
\hline \multirow{3}{*}{ Species } & \multicolumn{4}{|l|}{ Leaf } & \multicolumn{10}{|l|}{ Stem } \\
\hline & \multicolumn{2}{|l|}{ NG } & \multicolumn{2}{|l|}{ G } & \multicolumn{7}{|l|}{ NG } & \multicolumn{3}{|l|}{ G } \\
\hline & $\mathrm{A} 1$ & A2-i & G1 & G3 & $\mathrm{A} 1$ & $A 2-i$ & $\mathrm{~A} 2-\mathrm{ii}$ & B1 & B2 & B3 & C & G1 & $\mathrm{G} 2$ & G3 \\
\hline $\begin{array}{l}\text { L. aucheri ssp. aucheri } \\
\text { var. aucheri } 1\end{array}$ & $\begin{array}{l}55.33 \\
\pm 4.5\end{array}$ & $\begin{array}{l}0.00 \\
\pm 0.00\end{array}$ & $\begin{array}{l}0.00 \\
\pm 0.00\end{array}$ & $\begin{array}{l}10.5 \\
\pm 1\end{array}$ & $\begin{array}{l}0.00 \\
\pm 0.00\end{array}$ & $\begin{array}{l}350 \\
\pm 90.7\end{array}$ & $\begin{array}{l}0.00 \\
\pm 0.00\end{array}$ & $\begin{array}{l}0.00 \\
\pm 0.00\end{array}$ & $\begin{array}{l}0.00 \\
\pm 0.00\end{array}$ & $\begin{array}{l}0.00 \\
\pm 0.00\end{array}$ & $\begin{array}{l}0.00 \\
\pm 0.00\end{array}$ & $\begin{array}{l}0.00 \\
\pm 0.00\end{array}$ & $\begin{array}{l}0.00 \\
\pm 0.00\end{array}$ & $\begin{array}{l}0.00 \\
\pm 0.00\end{array}$ \\
\hline $\begin{array}{l}\text { L. aucheri ssp. aucheri } \\
\text { var. elegans }\end{array}$ & $\begin{array}{l}117.3 \\
\pm 4.48\end{array}$ & $\begin{array}{l}0.00 \\
\pm 0.00\end{array}$ & $\begin{array}{l}35 \\
\pm 8.65\end{array}$ & $\begin{array}{l}10 \\
\pm 0.00\end{array}$ & $\begin{array}{l}0.00 \\
\pm 0.00\end{array}$ & $\begin{array}{l}326.66 \\
\pm 43.15\end{array}$ & $\begin{array}{l}0.00 \\
\pm 0.00\end{array}$ & $\begin{array}{l}0.00 \\
\pm 0.00\end{array}$ & $\begin{array}{l}0.00 \\
\pm 0.00\end{array}$ & $\begin{array}{l}0.00 \\
\pm 0.00\end{array}$ & $\begin{array}{l}3 \\
\pm 0.8\end{array}$ & $\begin{array}{l}10 \\
\pm 4.00\end{array}$ & $\begin{array}{l}8 \\
\pm 2.5\end{array}$ & $\begin{array}{l}0.00 \\
\pm 0.00\end{array}$ \\
\hline $\begin{array}{l}\text { L. aucheri ssp. aucheri } \\
\text { var. kotschyanus }\end{array}$ & $\begin{array}{l}195 \\
\pm 72.23\end{array}$ & $\begin{array}{l}0.00 \\
\pm 0.00\end{array}$ & $\begin{array}{l}2.25 \\
\pm 0.55\end{array}$ & $\begin{array}{l}10 \\
\pm 0.00\end{array}$ & $\begin{array}{l}0.00 \\
\pm 0.00\end{array}$ & $\begin{array}{l}183.35 \\
\pm 21.24\end{array}$ & $\begin{array}{l}3 \\
\pm 0.00\end{array}$ & $\begin{array}{l}142.66 \\
\pm 8.35\end{array}$ & $\begin{array}{l}0.00 \\
\pm 0.00\end{array}$ & $\begin{array}{l}0.00 \\
\pm 0.00\end{array}$ & $\begin{array}{l}0.00 \\
\pm 0.00\end{array}$ & $\begin{array}{l}0.00 \\
\pm 0.00\end{array}$ & $\begin{array}{l}5.8 \\
\pm 1.2\end{array}$ & $\begin{array}{l}2 \\
\pm 0.5\end{array}$ \\
\hline $\begin{array}{l}\text { L.aucheri ssp. aucheri } \\
\text { var. tomentosus }\end{array}$ & $\begin{array}{l}223.33 \\
\pm 23.5\end{array}$ & $\begin{array}{l}0.00 \\
\pm 0.00\end{array}$ & $\begin{array}{l}9.8 \\
\pm 0.57\end{array}$ & $\begin{array}{l}10 \\
\pm 0.2\end{array}$ & $\begin{array}{l}120 \\
\pm 2.8\end{array}$ & $\begin{array}{l}138.66 \\
\pm 15.6\end{array}$ & $\begin{array}{l}0.00 \\
\pm 0.00\end{array}$ & $\begin{array}{l}0.00 \\
\pm 0.00\end{array}$ & $\begin{array}{l}0.00 \\
\pm 0.00\end{array}$ & $\begin{array}{l}0.00 \\
\pm 0.00\end{array}$ & $\begin{array}{l}0.00 \\
\pm 0.00\end{array}$ & $\begin{array}{l}18.75 \\
\pm 4.32\end{array}$ & $\begin{array}{l}1.9 \\
\pm 1\end{array}$ & $\begin{array}{l}0.00 \\
\pm 0.00\end{array}$ \\
\hline $\begin{array}{l}\text { L. aucheri ssp. aucheri } \\
\text { var. aucheri } 2\end{array}$ & $\begin{array}{l}140.67 \\
\pm 25.1\end{array}$ & $\begin{array}{l}0.00 \\
\pm 0.00\end{array}$ & $\begin{array}{l}0.00 \\
\pm 0.00\end{array}$ & $\begin{array}{l}10 \\
\pm 0.00\end{array}$ & $\begin{array}{l}0.00 \\
\pm 0.00\end{array}$ & $\begin{array}{l}367.66 \\
\pm 52.33\end{array}$ & $\begin{array}{l}0.00 \\
\pm 0.00\end{array}$ & $\begin{array}{l}25.24 \\
\pm 6.33\end{array}$ & $\begin{array}{l}0.00 \\
\pm 0.00\end{array}$ & $\begin{array}{l}0.00 \\
\pm 0.00\end{array}$ & $\begin{array}{l}0.00 \\
\pm 0.00\end{array}$ & $\begin{array}{l}15.5 \\
\pm 6.33\end{array}$ & $\begin{array}{l}0.00 \\
\pm 0.00\end{array}$ & $\begin{array}{l}0.00 \\
\pm 0.00\end{array}$ \\
\hline $\begin{array}{l}\text { L. aucheri ssp. aucheri } \\
\text { var. aucheri } 3\end{array}$ & $\begin{array}{l}0.00 \\
\pm 0.00\end{array}$ & $\begin{array}{l}282.33 \\
\pm 113.8\end{array}$ & $\begin{array}{l}1.5 \\
\pm 0.3\end{array}$ & $\begin{array}{l}30 \\
\pm 6.67\end{array}$ & $\begin{array}{l}0.00 \\
\pm 0.00\end{array}$ & $\begin{array}{l}250 \\
\pm 10.65\end{array}$ & $\begin{array}{l}0.00 \\
\pm 0.00\end{array}$ & $\begin{array}{l}0.00 \\
\pm 0.00\end{array}$ & $\begin{array}{l}0.00 \\
\pm 0.00\end{array}$ & $\begin{array}{l}0.00 \\
\pm 0.00\end{array}$ & $\begin{array}{l}0.00 \\
\pm 0.00\end{array}$ & $\begin{array}{l}5.5 \\
\pm 2.2\end{array}$ & $\begin{array}{l}0.00 \\
\pm 0.00\end{array}$ & $\begin{array}{l}4.5 \\
\pm 1.8\end{array}$ \\
\hline L. alutaceus & $\begin{array}{l}110 \\
\pm 35.61\end{array}$ & $\begin{array}{l}0.00 \\
\pm 0.00\end{array}$ & $\begin{array}{l}5.2 \\
\pm 1.20\end{array}$ & $\begin{array}{l}28.33 \\
\pm 3.2\end{array}$ & $\begin{array}{l}0.00 \\
\pm 0.00\end{array}$ & $\begin{array}{l}20 \\
\pm 7.67\end{array}$ & $\begin{array}{l}0.00 \\
\pm 0.00\end{array}$ & $\begin{array}{l}0.00 \\
\pm 0.00\end{array}$ & $\begin{array}{l}290.1 \\
\pm 48.88\end{array}$ & $\begin{array}{l}0.00 \\
\pm 0.00\end{array}$ & $\begin{array}{l}18.67 \\
\pm 3.5\end{array}$ & $\begin{array}{l}7.5 \\
\pm 3.67\end{array}$ & $\begin{array}{l}0.00 \\
\pm 0.00\end{array}$ & $\begin{array}{l}0.00 \\
\pm 0.00\end{array}$ \\
\hline L. cabulicus & $\begin{array}{l}60 \\
\pm 2.65\end{array}$ & $\begin{array}{l}0.00 \\
\pm 0.00\end{array}$ & $\begin{array}{l}0.00 \\
\pm 0.00\end{array}$ & $\begin{array}{l}26.33 \\
\pm 4.5\end{array}$ & $\begin{array}{l}0.00 \\
\pm 0.00\end{array}$ & $\begin{array}{l}293.33 \\
\pm 57.67\end{array}$ & $\begin{array}{l}0.00 \\
\pm 0.00\end{array}$ & $\begin{array}{l}0.00 \\
\pm 0.00\end{array}$ & $\begin{array}{l}0.00 \\
\pm 0.00\end{array}$ & $\begin{array}{l}0.00 \\
\pm 0.00\end{array}$ & $\begin{array}{l}1.9 \\
\pm 0.5\end{array}$ & $\begin{array}{l}1.2 \\
\pm 0.3\end{array}$ & $\begin{array}{l}0.00 \\
\pm 0.00\end{array}$ & $\begin{array}{l}2.66 \\
\pm 0.5\end{array}$ \\
\hline L. lasiocalyx & $\begin{array}{l}70.66 \\
\pm 3.55\end{array}$ & $\begin{array}{l}0.00 \\
\pm 0.00\end{array}$ & $\begin{array}{l}0.00 \\
\pm 0.00\end{array}$ & $\begin{array}{l}5.3 \\
\pm 1.5\end{array}$ & $\begin{array}{l}0.00 \\
\pm 0.00\end{array}$ & $\begin{array}{l}201.66 \\
\pm 53.2\end{array}$ & $\begin{array}{l}0.00 \\
\pm 0.00\end{array}$ & $\begin{array}{l}0.00 \\
\pm 0.00\end{array}$ & $\begin{array}{l}0.00 \\
\pm 0.00\end{array}$ & $\begin{array}{l}2 \\
\pm 0.00\end{array}$ & $\begin{array}{l}3 \\
\pm 0.00\end{array}$ & $\begin{array}{l}8.5 \\
\pm 2.33\end{array}$ & $\begin{array}{l}0.00 \\
\pm 0.00\end{array}$ & $\begin{array}{l}0.00 \\
\pm 0.00\end{array}$ \\
\hline L. macracanthus & $\begin{array}{l}55.33 \\
\pm 5.66\end{array}$ & $\begin{array}{l}0.00 \\
\pm 0.00\end{array}$ & $\begin{array}{l}15.8 \\
\pm 4.5\end{array}$ & $\begin{array}{l}10 \\
\pm 1.5\end{array}$ & $\begin{array}{l}185 \\
\pm 55.33\end{array}$ & $\begin{array}{l}276.67 \\
\pm 65.5\end{array}$ & $\begin{array}{l}0.00 \\
\pm 0.00\end{array}$ & $\begin{array}{l}0.00 \\
\pm 0.00\end{array}$ & $\begin{array}{l}0.00 \\
\pm 0.00\end{array}$ & $\begin{array}{l}0.00 \\
\pm 0.00\end{array}$ & $\begin{array}{l}0.00 \\
\pm 0.00\end{array}$ & $\begin{array}{l}5.8 \\
\pm 3.42\end{array}$ & $\begin{array}{l}0.00 \\
\pm 0.00\end{array}$ & $\begin{array}{l}0.00 \\
\pm 0.00\end{array}$ \\
\hline
\end{tabular}

Table 2. Continued.

\begin{tabular}{|c|c|c|c|c|c|c|c|c|}
\hline \multirow{3}{*}{ Species } & \multicolumn{8}{|l|}{ Calyx } \\
\hline & \multicolumn{6}{|l|}{ NG } & \multicolumn{2}{|l|}{ G } \\
\hline & $\mathrm{A} 1$ & $A 2-i$ & B1 & B3 & $\mathrm{C}$ & $\mathrm{D}$ & G1 & G3 \\
\hline $\begin{array}{l}\text { L. aucheri ssp. aucheri } \\
\text { var. aucheri } 1\end{array}$ & $\begin{array}{l}310 \\
\pm 77.33\end{array}$ & $\begin{array}{l}0.00 \\
\pm 0.00\end{array}$ & $\begin{array}{l}0.00 \\
\pm 0.00\end{array}$ & $\begin{array}{l}0.00 \\
\pm 0.00\end{array}$ & $\begin{array}{l}0.00 \\
\pm 0.00\end{array}$ & $\begin{array}{l}0.00 \\
\pm 0.00\end{array}$ & $\begin{array}{l}9 \\
\pm 3.7\end{array}$ & $\begin{array}{l}22.51 \\
\pm 7.8\end{array}$ \\
\hline $\begin{array}{l}\text { L. aucheri ssp. aucheri } \\
\text { var. elegans }\end{array}$ & $\begin{array}{l}202.66 \\
\pm 13.56\end{array}$ & $\begin{array}{l}0.00 \\
\pm 0.00\end{array}$ & $\begin{array}{l}75.2 \\
\pm 20\end{array}$ & $\begin{array}{l}0.00 \\
\pm 0.00\end{array}$ & $\begin{array}{l}0.00 \\
\pm 0.00\end{array}$ & $\begin{array}{l}24.5 \\
\pm 4\end{array}$ & $\begin{array}{l}7 \\
\pm 2\end{array}$ & $\begin{array}{l}23.84 \\
\pm 5.66\end{array}$ \\
\hline $\begin{array}{l}\text { L. aucheri ssp. aucheri } \\
\text { var. kotschyanus }\end{array}$ & $\begin{array}{l}633.33 \\
\pm 158.55\end{array}$ & $\begin{array}{l}0.00 \\
\pm 0.00\end{array}$ & $\begin{array}{l}0.00 \\
\pm 0.00\end{array}$ & $\begin{array}{l}0.00 \\
\pm 0.00\end{array}$ & $\begin{array}{l}17.67 \\
\pm 3.66\end{array}$ & $\begin{array}{l}0.00 \\
\pm 0.00\end{array}$ & $\begin{array}{l}17.6 \\
\pm 3.33\end{array}$ & $\begin{array}{l}45 \\
\pm\end{array}$ \\
\hline $\begin{array}{l}\text { L.aucheri ssp. aucheri } \\
\text { var. tomentosus }\end{array}$ & $\begin{array}{l}236.77 \\
\pm 27.6\end{array}$ & $\begin{array}{l}0.00 \\
\pm 0.00\end{array}$ & $\begin{array}{l}30.67 \\
\pm 12.33\end{array}$ & $\begin{array}{l}0.00 \\
\pm 0.00\end{array}$ & $\begin{array}{l}0.00 \\
\pm 0.00\end{array}$ & $\begin{array}{l}0.00 \\
\pm 0.00\end{array}$ & $\begin{array}{l}12.54 \\
\pm 4.47\end{array}$ & $\begin{array}{l}35 \\
\pm 3.6\end{array}$ \\
\hline $\begin{array}{l}\text { L. aucheri ssp. aucheri } \\
\text { var. aucheri } 2\end{array}$ & $\begin{array}{l}136 \\
\pm 14.53\end{array}$ & $\begin{array}{l}0.00 \\
\pm 0.00\end{array}$ & $\begin{array}{l}0.00 \\
\pm 0.00\end{array}$ & $\begin{array}{l}0.00 \\
\pm 0.00\end{array}$ & $\begin{array}{l}0.00 \\
\pm 0.00\end{array}$ & $\begin{array}{l}0.00 \\
\pm 0.00\end{array}$ & $\begin{array}{l}6.2 \\
\pm 3.33\end{array}$ & $\begin{array}{l}7.9 \\
\pm 2.1\end{array}$ \\
\hline $\begin{array}{l}\text { L. aucheri ssp. aucheri } \\
\text { var. aucheri } 3\end{array}$ & $\begin{array}{l}0.00 \\
\pm 0.00\end{array}$ & $\begin{array}{l}200 \\
\pm 35.7\end{array}$ & $\begin{array}{l}0.00 \\
\pm 0.00\end{array}$ & $\begin{array}{l}0.00 \\
\pm 0.00\end{array}$ & $\begin{array}{l}0.00 \\
\pm 0.00\end{array}$ & $\begin{array}{l}0.00 \\
\pm 0.00\end{array}$ & $\begin{array}{l}8.8 \\
\pm 2.2\end{array}$ & $\begin{array}{l}23.33 \\
\pm 5.3\end{array}$ \\
\hline L. alutaceus & $\begin{array}{l}122.3 \\
\pm 15.66\end{array}$ & $\begin{array}{l}0.00 \\
\pm 0.00\end{array}$ & $\begin{array}{l}0.00 \\
\pm 0.00\end{array}$ & $\begin{array}{l}223.33 \\
\pm 102.2\end{array}$ & $\begin{array}{l}0.00 \\
\pm 0.00\end{array}$ & $\begin{array}{l}0.00 \\
\pm 0.00\end{array}$ & $\begin{array}{l}5.5 \\
\pm 0.44\end{array}$ & $\begin{array}{l}25 \\
\pm 6.8\end{array}$ \\
\hline L. cabulicus & $\begin{array}{l}110.2 \\
\pm 21.33\end{array}$ & $\begin{array}{l}185.57 \\
\pm 28.63\end{array}$ & $\begin{array}{l}0.00 \\
\pm 0.00\end{array}$ & $\begin{array}{l}0.00 \\
\pm 0.00\end{array}$ & $\begin{array}{l}0.00 \\
\pm 0.00\end{array}$ & $\begin{array}{l}0.00 \\
\pm 0.00\end{array}$ & $\begin{array}{l}1 \\
\pm 0.3\end{array}$ & $\begin{array}{l}8.35 \\
\pm 4.45\end{array}$ \\
\hline L. Iasiocalyx & $\begin{array}{l}125 \\
\pm 18.88\end{array}$ & $\begin{array}{l}0.00 \\
\pm 0.00\end{array}$ & $\begin{array}{l}0.00 \\
\pm 0.00\end{array}$ & $\begin{array}{l}3 \\
\pm 1.5\end{array}$ & $\begin{array}{l}5 \\
\pm 2.33\end{array}$ & $\begin{array}{l}3 \\
\pm 0.9\end{array}$ & $\begin{array}{l}9 \\
\pm 3.25\end{array}$ & $\begin{array}{l}21.67 \\
\pm 5.00\end{array}$ \\
\hline L. macracanthus & $\begin{array}{l}210.15 \\
\pm 11.66\end{array}$ & $\begin{array}{l}200 \\
\pm 52.5\end{array}$ & $\begin{array}{l}0.00 \\
\pm 0.00\end{array}$ & $\begin{array}{l}0.00 \\
\pm 0.00\end{array}$ & $\begin{array}{l}0.00 \\
\pm 0.00\end{array}$ & $\begin{array}{l}0.00 \\
\pm 0.00\end{array}$ & $\begin{array}{l}22.2 \\
\pm 6.47\end{array}$ & $\begin{array}{l}33.33 \\
\pm 6.74\end{array}$ \\
\hline
\end{tabular}



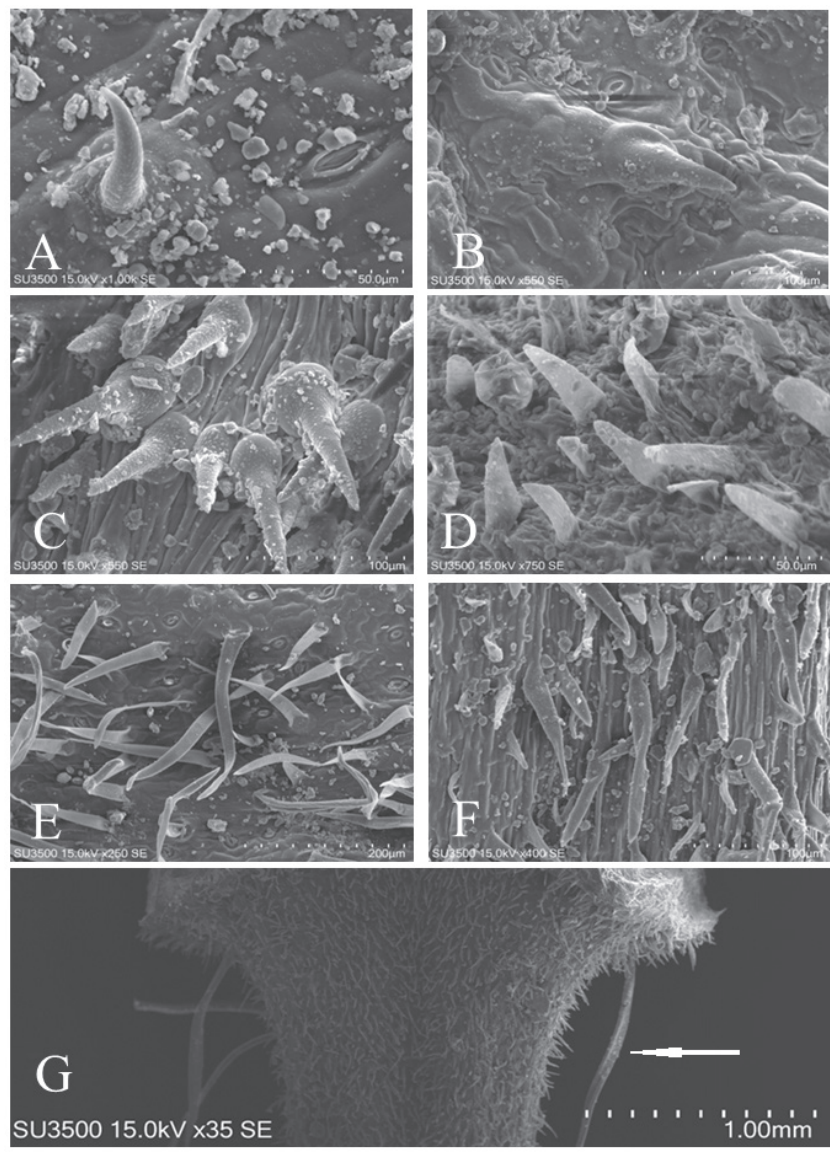

Figure 1. SEM micrographs of non-glandular unicellular trichomes in selected Lagochilus taxa. Prickles trichomes on the leaf (A-C). A: $L$. aucheri ssp. aucheri var. elegans. B: L. cabulicus. C: L. aucheri ssp. aucheri var. aucheri 2 . Short thin-walled trichomes (D-F). D: on the stem of $L$. macracanthus. E: on the calyx of $L$. aucheri ssp. aucheri var. aucheri 3. $F$ : on the stem of $L$. aucheri ssp. aucheri var. aucheri 1. Long thin-walled trichomes on the stem of $L$. aucheri ssp. aucheri var. kotschyanus (G).

the stems, leaves and calyces of $L$. aucheri ssp. aucheri var. aucheri 3 (Fig. 1E), and also on the stems of most specimens (e.g., L. lasiocalyx, L. aucheri ssp. aucheri var. aucheri 1 and L. aucheri ssp. aucheri var. aucheri 2, Fig. 1F). The stems of L. aucheri ssp. aucheri var. aucheri 2 and L. alutaceus had highest and lowest amounts of these types of trichomes, respectively (Table 2). Long thin-walled trichomes (ii): as long as 1000-2000 $\mu \mathrm{m}$ only on the stems of L. aucheri ssp. aucheri var. kotschyanus (Fig. 1G).

\section{Bicelled trichomes $(B)$.}

B1: short (50-200 $\mu \mathrm{m}$ in size), bicelled trichomes, densely covered by micropapillae; the apical cell is triangular and acute. This form was observed on the stems of L. aucheri ssp. aucheri var. kotschyanus and L. aucheri ssp. aucheri var. aucheri 2 (Fig. 2A) and on the calyces of L. aucheri ssp. aucheri var. elegans and L. aucheri ssp. aucheri var.
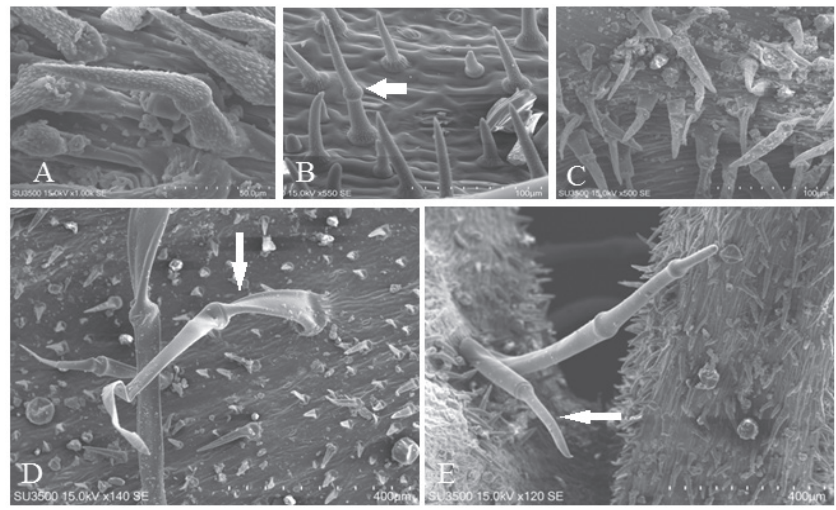

Figure 2. SEM micrographs of non-glandular trichomes in selected Lagochilus taxa. Bicelled trichomes type B1 on the stem of L. aucheri ssp. aucheri var. aucheri 2 (A) and on the calyx of L. aucheri ssp. aucheri var. elegans (B). Bicelled trichomes type B2 on the stem of $L$. alutaceus (C). Thin-walled bicelled trichomes type B3 on the calyx of $L$. alutaceus (D). Thick-walled bicelled trichomes type B3 on the stem of L. lasiocalyx (E).

tomentosus (Fig. 2B).

B2: short (50-200 $\mu \mathrm{m}$ in size), bi- celled trichomes, the basal cell is without micropapillae. This form can distinguishable on the stem of L. alutaceus (Fig. 2C).

B3. Long (1000-1500 $\mu \mathrm{m}$ in size), bicelled trichomes with rare micropapillae, this form was thin - walled on the L. alutaceus calyx (Fig. 2D) and thick-walled on $L$. lasiocalyx stem (Fig. 2E).

\section{Long, tricelled trichomes (1000-2000 $\mu \mathrm{m})(\mathrm{C})$.}

Elongated and thin-walled trichomes with rare micropapillae, can be observed on the stems of L. aucheri ssp. aucheri var. elegans, L. cabulicus and L. alutaceus (Fig. 3A-B), and thick-walled on the stems and calyces of L. lasiocalyx and L. aucheri ssp. aucheri var. kotschyanus, respectively (Fig. 2E and Fig. 3C).

\section{Long, four-celled trichomes (D).}

This type is found on the calyces of L. lasiocalyx and $L$. aucheri ssp. aucheri var. elegans (Fig. 3D-E).

\section{Glandular trichomes}

Three types of glandular trichomes were revealed by SEM micrographs, which all types were found in all studied specimens. The first type (G1) represented the sessile capitate glandular trichomes, (e.g., L. aucheri ssp. aucheri var. aucheri 1 and L. macracanthus; Fig. 3F). The second type (G2) represented the short-stalked capitate glandular trichomes (length of the stalk up to $10 \mu \mathrm{m}$ ) which were only registered on the stems of $L$. aucheri ssp. aucheri var. elegans, L. aucheri ssp. aucheri var. kotschyanus and L.aucheri ssp. aucheri var. tomentosus (Fig. 3G). The dominant glandular hairs, peltate glandular trichomes (G3), consist of a 

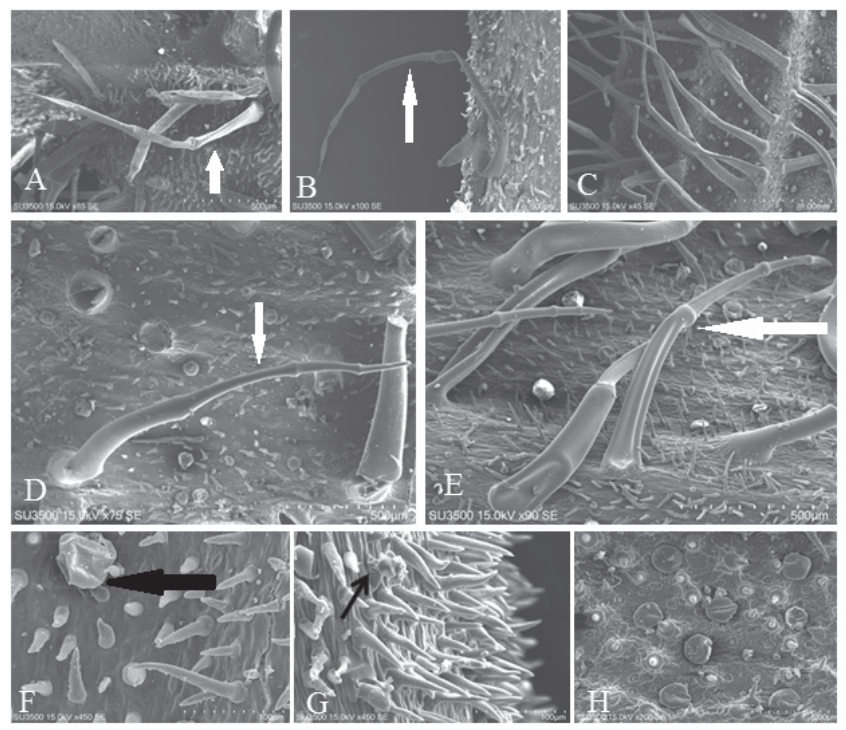

Figure 3. SEM micrographs of non-glandular and glandular trichomes in selected Lagochilus taxa. Long tricelled thin- or thick-walled trichomes of type C (A-C). Thin-walled trichomes on the stems L. alutaceus (A) and L. cabulicus (B). Thick-walled trichomes on the calyx of L. aucheri ssp. aucheri var. kotschyanus (C). Long, four-celled trichomes on the calyx of L. lasiocalyx (D) and L. aucheri ssp. aucheri var. elegans (E). Sessile capitate glandular trichomes on the calyx of $L$. aucheri ssp. aucheri var. aucheri 1. (F). Short stalked capitate glandular trichomes on the stem of L. aucheri ssp. aucheri var. tomentosus (G). Peltate glandular trichomes on the leaf of $L$. alutaceus $(H)$.
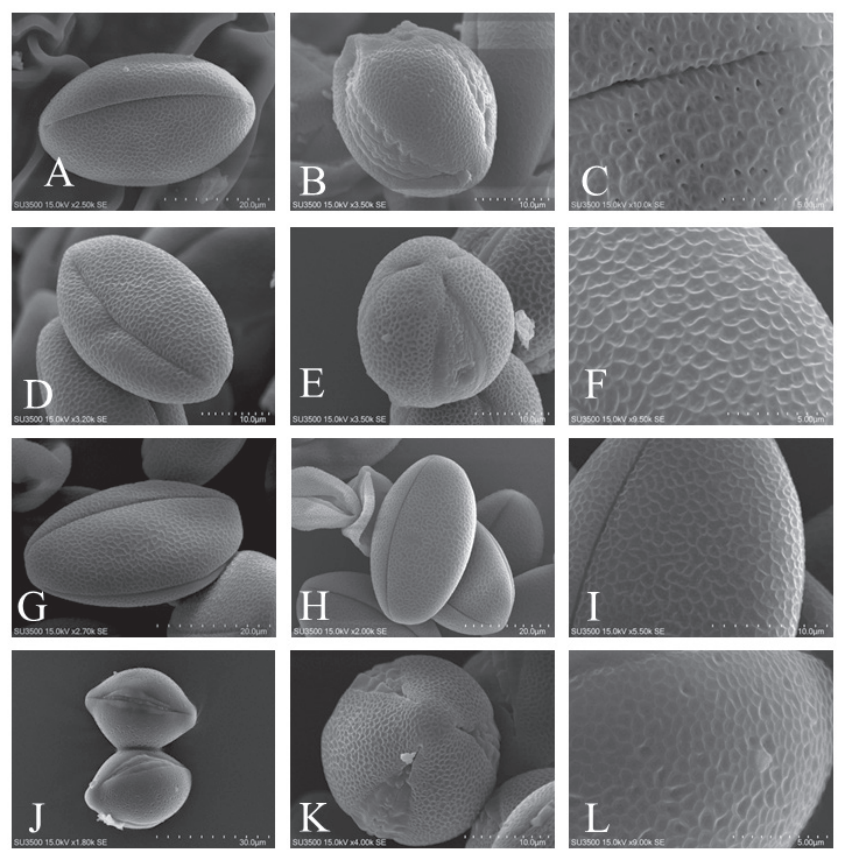

Figure 5. SEM micrographs of pollen grains in the Lagochilus taxa examined. A-C: L. macracanthus. D-F: L. aucheri ssp. aucheri var. kotschyanus. G-I: L. aucheri ssp. aucheri var. aucheri 1. J-L: L. aucheri ssp. aucheri var. aucheri 2.
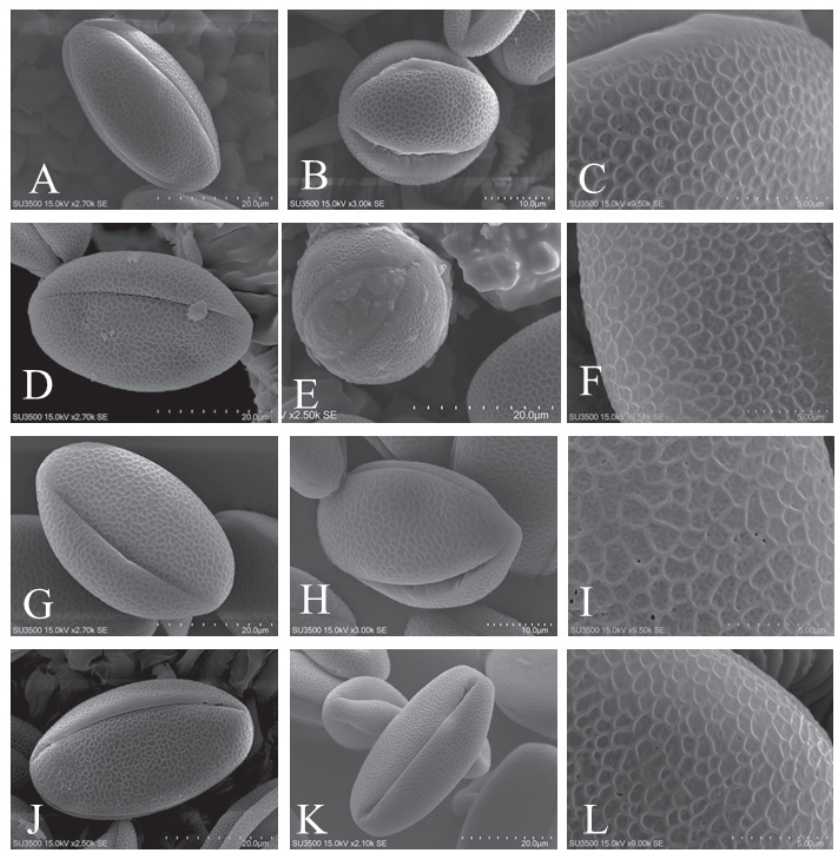

Figure 4. SEM micrographs of pollen grains in the Lagochilus taxa examined. A-C: L. alutaceus. D-F: L. cabulicus. G-I: L. aucheri ssp. aucheri var. elegans. J-L: L. aucheri ssp. aucheri var. aucheri 3.

basal cell, a short stalk cell and a multicellular head (4-8 cells). Its highest number was recorded on the leaves of L. aucheri ssp. aucheri var. aucheri $3, L$. cabulicus and $L$. alutaceus, and also on the calyx of L. aucheri ssp. aucheri var. kotschyanus (Table 2; Fig. 3H).

\section{General pollen features}

\section{Pollen size and shape}

The pollen grains were monads and mostly small to medium in size. The polar axis length varied from 29.35 $\mu \mathrm{m}$ in L. aucheri ssp. aucheri var. aucheri 2 to $43.91 \mu \mathrm{m}$ in L. aucheri ssp. aucheri var. aucheri 1 , while the equatorial diameter length varied from $17.6 \mu \mathrm{m}$ in $L$. aucheri ssp. aucheri var. kotschyanus to $24.59 \mu \mathrm{m}$ in L. aucheri ssp. aucheri var. aucheri 3 (Table 3). The shape of all pollen grains was prolate $(\mathrm{P} / \mathrm{E}=1.57-1.9)$ in equatorial view, except for $L$. aucheri ssp. aucheri var. aucheri 2 , which was prolate-subprolate $(\mathrm{P} / \mathrm{E}=1.34)$ (Table 3$)$. In polar view, the shape of all pollen grains was circular.

\section{Apertures}

Pollen grains were isopolar and tricolpate in all examined taxa. Simple colpi were elongated and narrowing at the poles. Colpus length ranges from $25.84 \mu \mathrm{m}$ in L. aucheri ssp. aucheri var. aucheri 2 to $41.46 \mu \mathrm{m}$ in L. aucheri ssp. aucheri var. aucheri 3 (Table 3). Mesocolpium value varied from $12.5 \mu \mathrm{m}$ in L. aucheri ssp. aucheri var. kotschyanus to 19.55 
Table 3. Summary of pollen morphological data for the Lagochilus taxa examined. All sizes are in $\mu \mathrm{m}$. Numbers refer to mean \pm standard deviation (minimum-maximum). Clg: colpus length. P: polar axis. E: equatorial axis. Sc: sculpturing type 1a, 1b, 1c = bireticulate; 2 : reticulate; 3 : microreticulate; 4: incomplete reticulate. All measurements are in $\mu \mathrm{m}$.

\begin{tabular}{|c|c|c|c|c|c|c|c|c|}
\hline Taxon & $\mathbf{P}$ & $\mathbf{E}$ & P/E & $\mathrm{Clg}$ & Mesocolpium & Apocolpium & Sc & Shape \\
\hline $\begin{array}{l}\text { L. aucheri ssp. aucheri } \\
\text { var. aucheri } 1\end{array}$ & $\begin{array}{l}43.91 \pm 1.7 \\
(40.9-46.83)\end{array}$ & $\begin{array}{l}23 \pm 2.2 \\
(20.17-26.26)\end{array}$ & 1.90 & $\begin{array}{l}41 \pm 1.9 \\
(37.65-43.19)\end{array}$ & $\begin{array}{l}15.68 \pm 1.41 \\
(14.36-17.95)\end{array}$ & $\begin{array}{l}4.2 \pm 1.04 \\
(3.69-5.41)\end{array}$ & 1c & Prolate \\
\hline $\begin{array}{l}\text { L. aucheri ssp. aucheri } \\
\text { var. elegans }\end{array}$ & $\begin{array}{l}41.31 \pm 1.24 \\
(35.68-42.94)\end{array}$ & $\begin{array}{l}22.8 \pm 1.42 \\
(20.93-25.18)\end{array}$ & 1.81 & $\begin{array}{l}38.51 \pm 1.06 \\
(37.05-40.04)\end{array}$ & $\begin{array}{l}16.53 \pm 1.8 \\
(15.21-17.85)\end{array}$ & $\begin{array}{l}4.35 \pm 1.7 \\
(5.7-6.2)\end{array}$ & $1 a$ & Prolate \\
\hline $\begin{array}{l}\text { L. aucheri ssp. aucheri } \\
\text { var. kotschyanus }\end{array}$ & $\begin{array}{l}33.08 \pm 1.41 \\
(31.22-34.29)\end{array}$ & $\begin{array}{l}17.6 \pm 0.62 \\
(16.97-18.16)\end{array}$ & 1.87 & $\begin{array}{l}30.56 \pm 1.64 \\
(29.50-32.44)\end{array}$ & $\begin{array}{l}12.5 \pm 1.36 \\
(10.75-15.3)\end{array}$ & $\begin{array}{l}3 \pm 0.72 \\
(2.4-4.03)\end{array}$ & $1 \mathrm{c}$ & Prolate \\
\hline $\begin{array}{l}\text { L. aucheri ssp. aucheri } \\
\text { var. tomentosus }\end{array}$ & $\begin{array}{l}39.82 \pm 0.91 \\
(38.57-40.98)\end{array}$ & $\begin{array}{l}22.95 \pm 0.97 \\
(21.21-24.35)\end{array}$ & 1.73 & $\begin{array}{l}37.81 \pm 1.15 \\
(35.5-39.49)\end{array}$ & $\begin{array}{l}15.61 \pm 2.29 \\
(13.69-18.72)\end{array}$ & $\begin{array}{l}4.7 \pm 0.45 \\
(4.04-5.13)\end{array}$ & 4 & Prolate \\
\hline $\begin{array}{l}\text { L. aucheri ssp. aucheri } \\
\text { var. aucheri } 2\end{array}$ & $\begin{array}{l}29.35 \pm 0.92 \\
(27.62-30.34)\end{array}$ & $\begin{array}{l}21.78 \pm 0.84 \\
(20.73-22.67)\end{array}$ & 1.34 & $\begin{array}{l}25.84 \pm 0.74 \\
(25.20-26.59)\end{array}$ & $\begin{array}{l}14 \pm 0.78 \\
(12.8-15.21)\end{array}$ & $\begin{array}{l}4.6 \pm 1.1 \\
(3.91-5.22)\end{array}$ & 2 & Prolate-subprolate \\
\hline $\begin{array}{l}\text { L. aucheri ssp. aucheri } \\
\text { var. aucheri } 3\end{array}$ & $\begin{array}{l}43.71 \pm 0.96 \\
(42.66-44.78)\end{array}$ & $\begin{array}{l}24.59 \pm 0.99 \\
(24.36-25.65)\end{array}$ & 1.77 & $\begin{array}{l}41.46 \pm 0.45 \\
(41.05-41.92)\end{array}$ & $\begin{array}{l}19.55 \pm 1 \\
(19.1-20.2)\end{array}$ & $\begin{array}{l}4.8 \pm 1.03 \\
(3.30-6.02)\end{array}$ & $1 a$ & Prolate \\
\hline L. alutaceus & $\begin{array}{l}35.59 \pm 2.27 \\
(33.12-39.93)\end{array}$ & $\begin{array}{l}19.21 \pm 1.81 \\
(15.46-21.70)\end{array}$ & 1.85 & $\begin{array}{l}31.56 \pm 2.09 \\
(30.16-35.07)\end{array}$ & $\begin{array}{l}14.72 \pm 1.35 \\
(13.15-15.47)\end{array}$ & $\begin{array}{l}2.45 \pm 0.21 \\
(2.34-2.78)\end{array}$ & $1 a$ & Prolate \\
\hline L. cabulicus & $\begin{array}{l}38.96 \pm 1.38 \\
(36.30-40.77)\end{array}$ & $\begin{array}{l}21.74 \pm 1.71 \\
(19.36-23.03)\end{array}$ & 1.79 & $\begin{array}{l}36.57 \pm 0.23 \\
(36.52-36.85)\end{array}$ & $\begin{array}{l}16.26 \pm 0.96 \\
(15.53-17)\end{array}$ & $\begin{array}{l}4.15 \pm 0.98 \\
(3.5-4.8)\end{array}$ & $1 a$ & Prolate \\
\hline L. lasiocalyx & $\begin{array}{l}33.73 \pm 1.54 \\
(30.03-36.14)\end{array}$ & $\begin{array}{l}21.43 \pm 1.5 \\
(19.08-23.64)\end{array}$ & 1.57 & $\begin{array}{l}31.2 \pm 1.62 \\
(29.38-34.57)\end{array}$ & $\begin{array}{l}16.13 \pm 1.17 \\
(15-18.11)\end{array}$ & $\begin{array}{l}3.2 \pm 0.83 \\
(2.5-3.92)\end{array}$ & 3 & Prolate \\
\hline L. macracanthus & $\begin{array}{l}39.83 \pm 0.74 \\
(39.08-40.69)\end{array}$ & $\begin{array}{l}23.66 \pm 1.5 \\
(22.42-24.51)\end{array}$ & 1.68 & $\begin{array}{l}37.78 \pm 0.95 \\
(36.05-39.98)\end{array}$ & $\begin{array}{l}16.03 \pm 1.73 \\
(13.75-18.06)\end{array}$ & $\begin{array}{l}4.85 \pm 0.63 \\
(4.11-5.63)\end{array}$ & $1 b$ & Prolate \\
\hline
\end{tabular}

$\mu \mathrm{m}$ in L. aucheri ssp. aucheri var. aucheri 3. Apocolpium diameter ranges from $2.45 \mu \mathrm{m}$ in L. alutaceus to $4.85 \mu \mathrm{m}$ in L. macracanthus (Table 3).

\section{Exine ornamentation}

Exine sculpture displayed four distinct types of surface ornamentation: bireticulate or suprareticulate (Fig. 4AL, Fig. 5A-F, and Table 3), reticulate (Fig. 5G-L, Table 3), microreticulate (Fig. 6A-C, Table 3) and incomplete reticulate (Fig. 6D-F, Table 3). Bireticulate patterns can be subdivided into three subtypes, based on the detailed configuration of the exine ornamentation patterns.

Bireticulate sculpture pattern. The most common (seven taxa) sculpture pattern among the studied taxa was the bireticulate sculpture pattern (special type of reticulate ornamentation, where a two-layered reticulum consisting of a suprareticulum supported by a microreticulate layer). According to the number of perforations per $25 \mu \mathrm{m}^{2}$, it can be divided into three subtypes (a-c). In subtype 1a, the number of perforations was $<5$ (L. alutaceus, $L$. cabulicus, $L$. aucheri ssp. aucheri var. elegans and $L$. aucheri ssp. aucheri var. aucheri 3 , Fig. 4A-L). In subtype $1 \mathrm{~b}$, the number of perforations was $>5$ (Fig. 5A-C, L. macracanthus). Bireticulate subtype $1 \mathrm{c}$ was determined with prolonged primary lumina without perforations, which was reported from L. aucheri ssp. aucheri var. kotschyanus (Fig. 5D-F) and $L$.
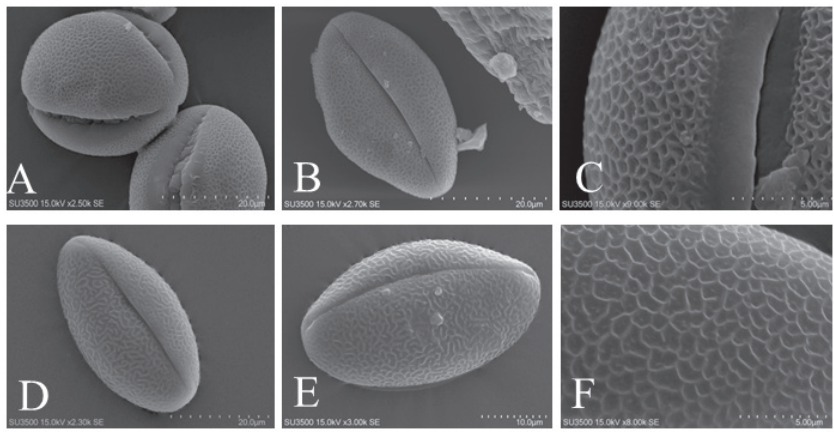

Figure 6. SEM micrographs of pollen grains in the Lagochilus taxa examined. A-C: L. lasiocalyx. D-F: L. aucheri ssp. aucheri var. tomentosus.

aucheri ssp. aucheri var. aucheri 1 (Fig. 5G-I).

Reticulate sculpture pattern. Among the examined taxa, L. aucheri ssp. aucheri var. aucheri 2 had reticulate sculpture pattern (Fig. 5J-L).

Microreticulate sculpture pattern. The microreticulate sculpture pattern is observed only in L. lasiocalyx (Fig. $6 \mathrm{~A}-\mathrm{C})$. In this pattern diameter of lumina is smaller than $1 \mu \mathrm{m}$.

Incomplete reticulate sculpture pattern. Incomplete reticulate sculpture pattern (network like pattern formed by exine elements, is incomplete), occurs for $L$. aucheri ssp. aucheri var. tomentosus (Fig. 6D-F). 


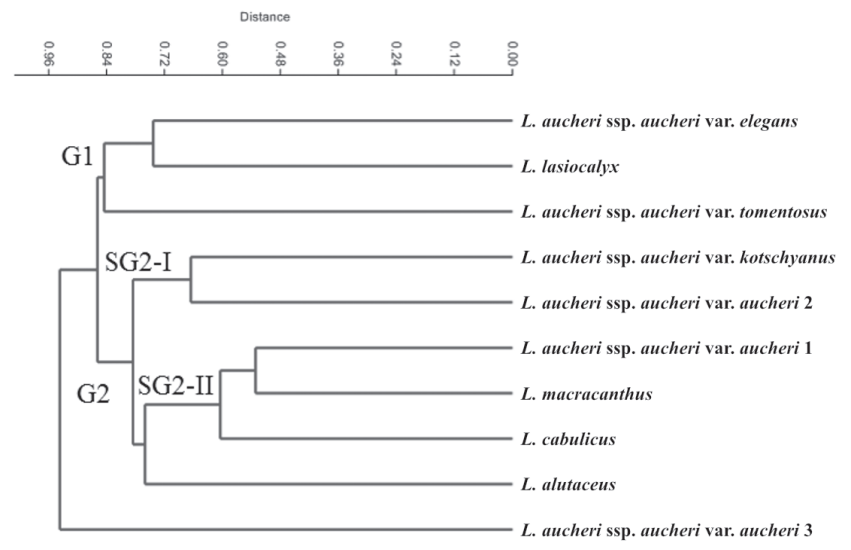

Figure 7. UPGMA dendrogram of the studied Lagochilus taxa based on micromorphological and palynological characteristics. G: group. SG: subgroup.

\section{Statistical analysis}

The UPGMA dendrogram based on the micromorphological and palynological features showed the existence of two main clusters (Fig. 7). Group1 (G1) contained specimens with three- or four- celled trichomes on the stems or calyces (L. lasiocalyx, L. aucheri ssp. aucheri var. elegans and L. aucheri ssp. aucheri var. tomentosus), while Group 2 (G2) was subdivided into two subgroups: subgroup I (SG2-I) contained specimens with two-celled trichomes of type B1 (L. aucheri ssp. aucheri var. aucheri 2 and L. aucheri ssp. aucheri var. kotschyanus). Subgroup II (SG2-II) contained specimens with short unicellular thin-walled trichomes on the stems and calyces (L. aucheri ssp. aucheri var. aucheri 1, L. macracanthus, L. cabulicus and L. alutaceus). L. aucheri ssp. aucheri var. aucheri 3 was sister to all Lagochilus species (Fig. 7). As seen in Figure 7, known varieties of $L$. aucheri were distributed through the tree.

The ANOVA result did not show significant differences $(p \leq 0.05)$ in the number between all observed types of trichomes, however, PCA-biplot showed that some of species had distinct type of trichomes that were useful in identification of them (Fig. 8). For example, the number of trichomes type $\mathrm{A} 1$ on the calyces is a distinguishing trait for identification of $L$. aucheri ssp. aucheri var. kotschyanus, however, L. aucheri ssp. aucheri var. tomentosus were identified by highest number of trichomes of type A1on the leaves. In addition, trichomes of type $\mathrm{B} 2$ and $\mathrm{C}$ on the stems was significant trait for L. alutaceus.

\section{Discussion}

\section{Micromorphological characters}

Both glandular and non-glandular trichomes were ex- isted on the stems, leaves and calyces of all investigated taxa. The trichomes types presented on the studied species were mostly similar to those of family Lamiaceae reported by Navarro and El Qualidi (2000), Cantino (1990) and Atalay et al. (2016a). Generally, number of non-glandular trichomes was higher than glandular ones, which is a common feature in Lamiaceae family (Metcalfe and Chalk 1950).

Our study showed that the size and cell number of trichomes were valuable and could be applied to classify the non-glandular trichomes into four types including 10 forms; therefore, they can be used as taxonomic tools in species and intraspecific identification.

Two main types of glandular trichomes were capitate and peltate. The studied taxa had two types of capitate trichomes which differed from each other in their stalk length. Talebi and Rezakhanlou (2012) reported the capitate trichomes with a basic and two apical cells in $L$. macracanthus. The peltate trichomes of Lamiaceae have mostly a secretory head of four central and 6-14 peripheral cells (Werker 1993). Peltate glandular hairs with eightcelled head were previously reported in L. macracanthus (Talebi and Rezakhanlou 2012). The distribution of glandular trichomes is obviously correlated with their role in pollination (Navarro and El Qualidi, 2000). In Lagochilus genus, this type totally appears on the outer side of the calyces and sparse on the stems.

\section{Pollen grain morphology}

Erdtman (1945) classified the Lamiaceae family into two subfamilies based on the number of apertures and nuclei in the mature pollen grains. The first group comprises the subfamily Lamioideae with tricolpate pollen grains and the second group contains the subfamily Nepetoideae characterized by hexacolpate pollen grains. According to the latest molecular phylogenetic studies by Bendiksby et al. (2011) the genus Lagochilus, included in the subfamily Lamioideae and tribe Leonureae. The present study showed that the all the examined taxa have tricolpate pollen grains similar with the other genera of subfamily Lamioideae (Abu-Asab and Cantino 1994; Atalay et al. 2016b).

The shapes of the pollen grains in equatorial view is prolate except for $L$. aucheri ssp. aucheri var. aucheri 2 , with prolate-subprolate shape $(\mathrm{P} / \mathrm{E}=1.34)$. The state of hydration and/or fixation could be affecting the pollen shape (Demissew and Harley 1992; León-Arencibia and La-Serna Ramos 1992; Moon et al. 2008a). Thus, differences in shape among the pollen grains may not be significant or even applicable in their taxonomy (Xiang et al. 2013). Moon et al. (2008a) suggested, in order preserving a more natural form, careful processing such as critical point drying of the fresh material is required. 
The observed exine sculpture patterns in Lagochilus were determined as bireticulate, reticulate, microreticulate and also incomplete reticulate. Reticulate and microreticulate exins are common in several species of subfamily Lamioideae and in other genera of Lamiaceae (Wagstaff 1992; Abu-Asab and Cantino 1992, 1994; Çelenk et al. 2008 a,b; Moon et al. 2008 a,b,c; Özler et al. 2011, 2013; Atalay et al. 2016b). Bireticulate exin are a plesiomorphic in subfamily Lamioidae, while it is apomorphic in Lamiaceae (Cantino 1992 a,b). Derived states as psilate, granulate, rugulate and suprareticulate-rugulate forms of sculpturing pattern and branched columellae occur in some members of Lamioids. Similar pollen features suggest relationships within and between certain genera in Lamioidae (Abu-Asab and Cantino 1994; Atalay et al. 2016b). Incomplete reticulate sculpture pattern occured for L.aucheri ssp. aucheri var. tomentosus (Fig. 6D-F). This pattern not been reported previously in Lamiaceae.

Among the investigated taxa, palynological properties of $L$. aucheri have been provided by Abu-Asab and Cantino (1994). Characteristics investigated by them correlate with our current results, except for the $\mathrm{P} / \mathrm{E}$ ratios, which are possibly due to differences in procedure of preparation.

\section{Systematic significance of trichome and pollen grains micromorphology}

\section{L. aucheri complex}

Jamzad (1988), in her taxonomic revision of the genus Lagochilus in Iran, recognized two subspecies for L. aucheri: ssp. heterophyllus and ssp. aucheri. Moreover, she identified four varieties for ssp. aucheri. She stated that the indumentum is one of the characteristics that can be used in distinguishing varieties. Results of our investigation showed that the trichomes micromorphology can be used in distinguishing varieties that presented by Jamzad (1988). She has reported that $L$. aucheri ssp. aucheri var. aucheri was characterized by glabrous stem and calyx, while we observed short unicellular trichomes on the stem and calyx of this taxon. Moreover, leaves of this variety had lowest number of prenominated trichomes.

L. kotschyanus Boiss. was originally described as a species but was reduced to a variety of $L$. aucheri by Bornmüller (1907). L. aucheri ssp. aucheri var. kotschyanus has long unicellular trichomes on the stems and tricelled trichomes on the calyces, which were not observed in any other varieties of the species.

L. aucheri ssp. aucheri var. elegans and $L$. aucheri ssp. aucheri var. tomentosus, are two new infraspecific taxa that were described by Jamzad (1988). In our study, L. aucheri ssp. aucheri var. elegans was characterized by four-celled trichomes on the calyces, and L. aucheri ssp. aucheri var. tomentosus was identified by short unicellular trichomes

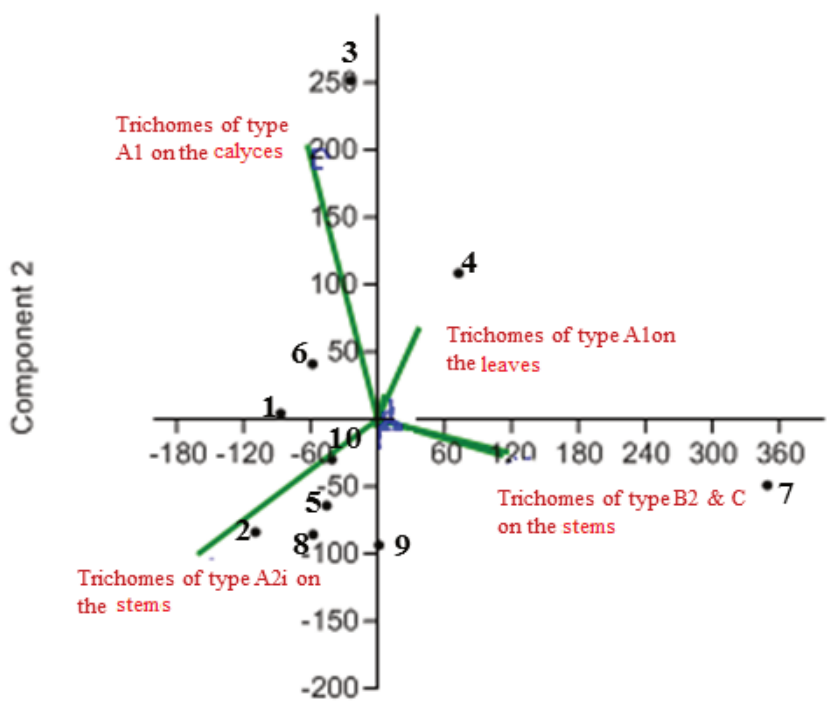

Component 1

Figure 8. PCA-biplot of the studied taxa and their trichome features. 1: L. aucheri ssp. aucheri var. aucheri 1. 2: L. aucheri ssp. aucheri var. elegans. 3: L. aucheri ssp. aucheri var. kotschyanus. 4: L. aucheri ssp. aucheri var. tomentosus. 5: L. aucheri ssp. aucheri var. aucheri 2. 6: L. aucheri ssp. aucheri var. aucheri 3. 7: L. alutaceus. 8: L. cabulicus. 9: L. lasiocalyx. 10: L. macracanthus.

on the stems and bicelled trichomes on the calyces. In addition, $L$. aucheri ssp. aucheri var. tomentosus identified by highest number of trichomes of type Alon the leaves.

In carrying out of determination of specimens, we identified plants which have basic traits of L.aucheri but differed from known species in some morphological characteristics such as ratio of teeth length /calyx tube or calyx teeth numbers. This holds true for plants of Zanjan (L. aucheri ssp. aucheri var. aucheri 2$)$ and Dizin ( $L$. aucheri ssp. aucheri var. aucheri 3) populations. Detailed micromorphological analysis revealed that trichomes of type A1 were observed on the leaves and calyces of all specimens, except $L$. aucheri ssp. aucheri var. aucheri 3 , while, unicellular thin-walled trichomes with 200-300 $\mu \mathrm{m}$ in size (A2 i type), present only on the leaves, stems and calyces of L. aucheri ssp. aucheri var. aucheri 3 . Similarly, bicelled trichomes of type B1 were observed on the stems of $L$. aucheri ssp. aucheri var. aucheri 2 and $L$. aucheri ssp. aucheri var. kotschyanus, however, latter vary from $L$. aucheri ssp. aucheri var. aucheri 2 in having trichomes of type A2ii on the stems and trichomes of type $\mathrm{C}$ on the calyces. The basic shape of the pollen grains in most taxa was prolate, however prolate-subprolate pollen grains was recorded for L. aucheri ssp. aucheri var. aucheri 2 . Moreover, reticulate sculpturing pattern of pollen grains were observed only in L. aucheri ssp. aucheri var. aucheri 
2. L. aucheri ssp. aucheri var. aucheri 3 had highest value of colpus length and mesocolpium area among all taxa examined. Therefore, it seems to be new intraspecific ranks in L. aucheri complex that need to additional and detailed morphological and molecular studies.

In our UPGMA tree of micromorphological and palynological characteristics, the examined taxa are separated from each other, but varieties of L. aucheri do not grouped together and not nested in the same clade as the members of $L$. aucheri. Therefore, L. aucheri seems to be a polyphyletic and this genus needs to revise of morphological characteristics for identifying of species.

\section{Other Lagochilus species}

Jamzad (1988) described L. lasiocalyx as the new combination, based on L. aucheri var. lasiocalyx Stapf., and treated $L$. aucheri var. perhispidus Bornm. as a synonym of L. lasiocalyx. In flora of Iran (Jamzad 2012), L. lasiocalyx has placed toward known varieties of $L$. aucheri. Result of UPGMA tree of micromorphological characteristics corroborates her morphology-based conclusion; L. lasiocalyx showed a close relationship with L. aucheri ssp. aucheri var. elegans and L. aucheri ssp. aucheri var. tomentosus.

According to Jamzad (1988), there was close affinity and the high degree of morphological similarities between L. cabulicus and L. aucheri, in so far as it is difficult to distinguish them. We could differentiate them by trichomes and pollen grains characteristics. The trichomes size on the leaves of $L$. cabulicus was very short $(<50 \mu \mathrm{m})$ while it varied from 100-200 $\mu \mathrm{m}$ in L. aucheri 1, also we could differentiate them by the shorter size of the pollen grains in L. cabulicus. The polar and equatorial axis ranged from 36.30-40.77 $\mu \mathrm{m}$ and 19.36-23.03 $\mu \mathrm{m}$, respectively in $L$. cabulicus, while they varied from $40.9-46.83 \mu \mathrm{m}$ and $20.17-$ $26.26 \mu \mathrm{m}$ in L. aucheri 1. Despite bireticulate sculpture pattern observed in both species, they varied from each other according to the number of perforations per $25 \mu \mathrm{m}^{2}$.

In the UPGMA tree, a close relationship could be identified among L. cabulicus, L. macracanthus and L. alutaceus, which these results, generally were corroborate with Rechinger classification (1982).

The result of statistical analysis showed that trichomes characteristics such as density and cell numbers of nonglandular trichomes could be used as a relevant features for identification of the investigated taxa concerning they stable position between studied populations of each species.

\section{Key to studied taxa according to non-glandular tri- chomes}

Based on our results of trichomes morphology and density of Lagochilus species, we proposed that the type of indumentum can be used as a significant trait for iden- tification of the examined taxa. Among non-glandular trichomes, size, cell number and density are considered as valuable, therefore, a key to the studied taxa of Lagochilus according to the result of the non-glandular trichomes are given below.

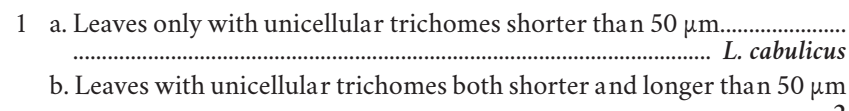

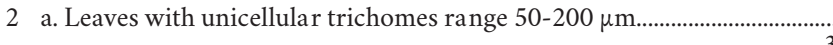

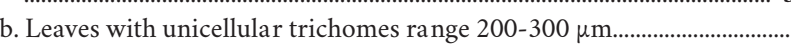
…................................................. L. aucheri $\mathrm{ssp}$. aucheri var. aucheri 3

3 a. Stems only with unicellular trichomes 4

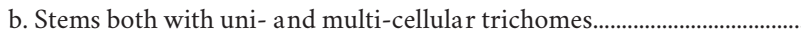

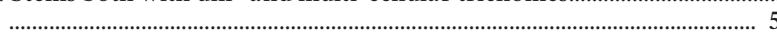

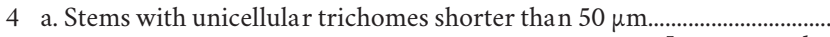

......................................................................................... L. macracanthus

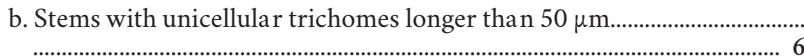

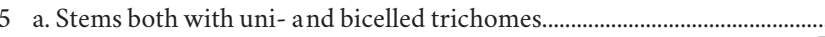

.

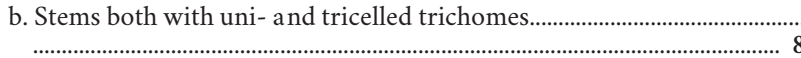

6 a. Calyces with short unicellular trichomes up to $100 \mu \mathrm{m}$...

.................................................... L. aucheri ssp. aucheri var. aucheri 1

b. Calyces with bicelled trichomes range 100-200 $\mu \mathrm{m} . .$. L. aucheri ssp. aucheri var. tomentosus

7 a. Stems with short unicellular trichomes $(50-200 \mu \mathrm{m})$, calyces only with unicellular trichomes.

L. aucheri ssp. aucheri var. aucheri 2

b. Stems with long unicellular trichomes (1000-2000 $\mu \mathrm{m})$, calyces with uni- and tricelled trichomes

L. aucheri ssp. aucheri var. kotschyanus

8 a. Calyces with uni- and bicelled trichomes.

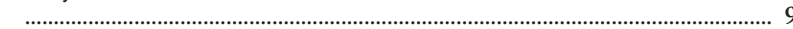

b. Calyces with uni- and tricelled trichomes.. L. lasiocalyx

9 a. Calyces with bicelled trichomes range 500-1000 $\mu \mathrm{m}$, without four-celled trichomes.

L. alutaceus

b. Calyces with bicelled trichomes ranges 100-200 $\mu \mathrm{m}$, with four-celled trichomes. L. aucheri ssp. aucheri var. elegans

\section{Conclusion}

According to the Plant List (Govaerts et al. 2013), some of species and infraspecific taxa currently recognized as synonyms (e.g., L. lasiocalyx, , L. aucheri ssp. aucheri var. elegans and L.aucheri ssp. aucheri var. tomentosus), while, based on micromorphological results were presented here, all known species and infraspecific taxa were delimited.

In conclusion, the present study provides micromorphological and palynological characteristics of the genus Lagochilus that would be applicable in identification, 
delimitation and classification of the genus; however, Phylogenetic and morphological studies of Lagochilus, based on complete sampling will be necessary to confirm existence of new taxa and to illuminate the intraspecific relationships of the genus.

\section{Acknowledgements}

We would like to thank Mr. A. Javadi Anaghizi (Central Laboratory of the Shahid Beheshti University) for his help in SEM.

\section{References}

Abu-Asab MS, Cantino PD (1989) Pollen morphology of Trichostema (Labiatae) and its sytematic implications. Syst Bot 14:359-369.

Abu-Asab MS, Cantino PD (1992) Pollen morphology in subfamily Lamioideae (Labiatae) and its phylogenetic implications. In Harley RM, Reynolds T, Eds., Advances in Labiatae Science. Royal Botanic Gardens, Kew, pp. 97-112.

Abu-Asab MS, Cantino PD (1993a) Phylogenetic implications of pollen morphology in tribe Ajugeae (Labiatae). Syst Bot 18:100-122.

Abu-Asab MS, Cantino PD (1993b) Systematic implications of pollen morphology in tribe Prostanthereae (Labiatae). Syst Bot 18:563-574.

Abu-Asab MS, Cantino PD (1994) Systematic implications of pollen morphology in subfamilies Lamioideae and Pogostemonoiedae (Labiatae). Ann Missouri Bot Gard 81:635-686.

Atalay Z, Celep F, Bara F, Doğan M (2016a) Systematic significance of anatomy and trichome morphology in Lamium (Lamioideae; Lamiaceae). Flora 22:60-75.

Atalay Z, Celep F, Bara F, Doğan M (2016b) Pollen morphology of the genus Lamium L. (Lamiaceae) and its systematic implications. Flora 21:68-84.

Badamtsetseg B, Myoung LS, Yuon LH (2012) Pollen morphology of the family Lamiaceae in Mongolia.J Korean Nat 5:169-179.

Bendiksby M, Thorbek LB, Scheen AC, Lindqvist C, Ryding $\mathrm{O}$ (2011) An updated phylogeny and classification of Lamiaceae subfamily Lamioideae. Taxon 60:471-484.

Boissier E (1879) Flora Orientalis. Vol. 4, 768-77 L. Basiliae \& Genevae.

Boissier E (1848) In De Candolle AP, Ed, Prodromus Systematis Naturalis Regni Vegetabilis, Vol. 12. Victor Masson, Paris, 515.

Bornmüller JFN (1907) Plantae Straussianae - sive enumera- tio plantarum a Th. Strauss annis 1889-1899 in Persia occidentali collectarum. Pars III. Beih Bot Centralbl, Abt. 22:134.

Cantino PD (1990) The phylogenetic significance of stomata and trichomes in the Labiatae and Verbenaceae. J Arnold Arbor 71:323-370.

Cantino PD (1992a) Evidence for a polyphyletic origin of the Labiatae. Ann Missouri Bot Gard 79:361-379.

Cantino PD (1992b) Toward a phylogenetic classification of the Labiatae. In Harley RM, Reynolds T, Eds, Advances in Labiatae Science. Royal Botanic Gardens, Kew, pp 27-37.

Çelenk S, Tarimcilar G, Biçakçi A, Kaynak G, Malyer H (2008a) A palynological study of Mentha L. Bot J Linn Soc 157:141-154.

Çelenk S, Dirmenci T, Malyer H, Biçakçi A (2008b) A palynological study of the genus Nepeta L. (Lamiaceae). Plant Syst Evol 276:105-123.

Chizhov OS, Kessenikh AV, Yakovlev IP, Zolotarev BM, Petukhov VA (1970) Bull Acad Sci USSR, Div Chem Sci 19:1866.

Demissew SD, Harley MM (1992) Trichome, seed surface and pollen characters in Stachys (Lamioideae: Labiatae) in tropical Africa. In Harley RM, Reynolds T, Eds., Advances in Labiatae Science. Royal Botanical Gardens, London, Kew, pp 149-166.

Doaigey AR (1991) Trichome types in the genus Otostegia Benth. (Lamiaceae). J King Saud Univ Sci 3:23-30.

Erdtman G (1945) Pollen morphology and plant taxonomy. IV. Labiatae,Verbenaceae, and Avicenniaceae. Svensk Bot Tidskr 39:279-285.

Faegri K, Iversen J (1975) Textbook of Pollen Analysis, 3rd ed. Munksgaard, Copenhagen.

Firdous S, Habib A, Manzoor H, Muqarrab S (2015) Pollen morphology of Ajuga L., Lamium L. and Phlomis L. (Lamiaceae) from district Abbottabad Pakistan. Pak J Bot 47:269-274.

Gairola S, Naidoo Y, Bhatt A, Nicholas A (2009) An investigation of the foliar trichomes of Tetradenia riparia (Hochst.) Codd [Lamiaceae]: an important medicinal plant of Southern Africa. Flora 204:325-330.

Gonzales WL, Negrittoa MA, Suarez LH, Gianoli E (2008) Induction of glandular and non-glandular trichomes by damage in leaves of Madia sativa under contrasting water regimes. Acta Oecol 33:128-132.

Govaerts R, Paton A, Harvey Y, Navarro T (2013) World checklist of Lamiaceae and Verbenaceae. Kew, Richmond: The Board of Trustees of the Royal Botanic Gardens. http://www.kew.org/wcsp/lamiaceae/.

Harley MM, Paton A, Harley RM, Cade PG (1992) Pollen morphological studies in tribe Ocimeae (Nepetoideae: Labiatae): I. Ocimum L. Grana 31:161-176.

Harley RM, Atkinson S, Budantsev AL, Cantino PD, Conn BJ, Grayer R, Harley MM, Dekok R, Krestovskaja T, 
Morales R, Paton AJ, Ryding O, Upson T (2004) Labiatae. In Kadereit JW, Ed., The Families and Genera of Vascular Plants. Vol. 7. Springer, Berlin, pp. 167-275.

Hassan N, Osman AK, El Garf IA (2009) Pollen types of the Egyptian species of the genus Salvia (Lamiaceae). Feddes Repert 120:394-404.

Hesse M, Halbritter H, Zetter R, Weber M, Buchner R, Frosch-Radivo A, Ulrich S (2009) Pollen Terminology - An Illustrated Handbook. Springer, Wien, New York.

Ikramov MI (1976) Rod Lagochilus Srednej Azii. Izdat Taskent, Izdat Fan Uzbekskoj SSR.

Jalili A, Jamzd Z (1999) Red Data Book of Iran. Research Institute of Forests and Rangelands, Tehran, Iran.

Jamzad Z (1988) The genus Lagochilus (Labiatae) in Iran. Iranian J Bot 4:91-103.

Jamzad Z (2012) Lamiaceae. In Assadi M, Maassoumi A, Mozaffarian V, Eds., Flora of Iran. Vol. 76. Research Institute of Forests and Rangelands, Tehran (in Persian), pp 337-349.

Jiao Y, Zhang CG, Zhang T, Chou GX, Xu H LQ (2014) Antiinflammatory effects of aqueous extracts of whole herbs from five Lagochilus species in vitro. Chinese Journal of New Drugs and Clinical Remedies 33:204-211.

León-Arencibia MC, La-Serna Ramos IE (1992) Palynological study of Lavandula (sect. Pterostoechas, Labiatae): Canario-maderiense endemics. Grana 31:187-195.

Bini Maleci L, Servettaz O (1991) Morphology and distribution of trichomes in Italian species of Teucrium Sect. Chamaedrys (Labiatae) - a taxonomical evaluation. Plant Syst Evol 174:83-91.

Metcalfe CR, Chalk L (1950) Anatomy of the Dicotyledons. Vol. 2. Oxford Press, London.

Moon HK, Hong SP (2003) Pollen morphology of the genus Lycopus (Lamiaceae). Ann Bot Fenn 40:191-198.

Moon HK, Vinckier S, Smets E, Huysmans S (2008a) Comparative pollen morphology and ultrastructure of Mentheae subtribe Nepetinae (Lamiaceae). Rev Paleobot Palynol 149:174-186.

Moon HK, Vinckier S, Walker JB, Smets E, Huysmans S (2008b) A search for phylogenetically informative pollen characters in the subtribe Salviinae/Mentheae: Lamiaceae. Int J Plant Sci 169:455-471.

Moon HK, Vinckier S, Smets E, Huysmans S (2008c) Palynological evolutionary trends within the tribe Mentheae with special emphasis on subtribe Menthinae (Nepetoideae: Lamiaceae). Plant Syst Evol 275:93-108.

Navarro T, El Qualidi J (2000) Trichome morphology in Teucrium L. (Labiatae): a taxonomic review. Ann Bot Garden Madrid 57:277-297.

Özler H, Pehlivan S, Kahraman A, Doğan M, Celep F, Başer B, Yavru A, Bagherpour S (2011) Pollen morphology of the genus Salvia L. (Lamiaceae) in Turkey. Flora 206:316-327. Özler H, Pehlivan S, Celep F, Doğan M, Kahraman A, Fisne
AY, Başer B, Bagherpour S (2013) Pollen morphology of Hymenosphace and Aethiopis sections of the genus Salvia (Lamiaceae) in Turkey. Turk J Bot 37:1070-1084.

Parsa A (1949) Flora de I'Iran. Vol. 4:821-826.

Peter J, Shanower T (1998) Plant glandular trichomes chemical factories with many potential uses. Resonance 3:4145.

Podani J (2000) Introduction to the Exploration of Multivariate Biological Data. Backhuys Publisher, Leiden, p. 407.

Rechinger KH (1982) Flora Iranica. No. 150. Akademische Druck- u. Verlagsanstalt, Graz, pp. 337-344.

Salmaki Y, Jamzad Z, Zarre S, Brauchler C (2008) Pollen morphology of Stachys (Lamiaceae) in Iran and its systematic implication. Flora 203:627-639.

Salmaki Y, Zarre S, Jamzad Z, Bräuchler C (2009) Trichome micromorphology of Iranian Stachys (Lamiaceae) with emphasis on its systematic implication. Flora 20:371-381.

Taban S, Masoudi Sh, Chalabian F, Delnavaz B, Rustaiyan A (2009) Chemical composition and antimicrobial activities of the essential oils from flower and leaves of Lagochilus kotschyanus Boiss. A new species from Iran. J Med Plant 8:58-63.

Talebi SM, Rezakhanlou A (2012) Biological study of Lagochilus maracanthus Fish. et Mey. endemic species of Iran. J Chem Pharm Res 4:633-639.

Talebi SM, Mahdiyeh M, Ghorbani Nohooji M, Akhani M (2018a) Analysis of trichome morphology and density in Salvia nemorosa L. (Lamiaceae) of Iran. Botanica 24:49-58.

Talebi SM, Ghorbani Nohooji M, Yarmohammadi M, Azizi N, Matsyura A (2018b) Trichomes morphology and density analysis in some Nepeta species of Iran. Med Bot 39:51-62.

Wagner GJ (1991) Secreting glandular trichomes: more than just hairs. Plant Physiol 96:675-679.

Wagstaff SJ (1992) A phylogenetic interpretation of pollen morphology in tribe Mentheae (Labiatae). In Harley RM, Reynolds T, Eds., Advances in Labiatae Science. Royal Botanic Gardens, Kew, pp. 113-124.

Werker E (1993) Fuction of essential oil secreting glandular hairs in aromatic plants of the Lamiaceae. A review. Flavour Fragr J 8:249-255.

Wunderlich R (1967) Ein Vorschlag zu einer natürlichen Gliederung der Labiaten auf Grund der Pollenkörner, der Samenentwicklung und des reifen Samens. Österr Bot Z 114:383-483.

Xiang CL, Funamoto T, Evangelista EV, Zhangd Q, Penga H (2013) Pollen morphology of the East Asiatic genus Chelonopsis (Lamioideae: Lamiaceae) and allied genera, with reference to taxonomic implications and potential pollination ecology. Plant Biosys 147:620-628.

Xiao K, Mao X, Lin Y, Xu H, Zhu Y, Cai Q, Xie H, Zhang $\mathrm{J}$ (2017) Trichome, a functional diversity phenotype in plant. Mol Biol 6:1. DOI: 10.4172/2168-9547.1000183 
Zhang LM, Zeng XQ, Sanderson SC, Byalt VV, Sukhorukov AP (2017) Insight into Central Asian flora from the Cenozoic Tianshan montane origin and radiation of Lagochilus (Lamiaceae). PloS ONE 12(9):e0178389. 
